\title{
FORMAÇÃO PROFISSIONAL INICIAL EM ATIVIDADE FíSICA ADAPTADA: ANÁLISE DA PRODUÇÃO CIENTÍFICA INTERNACIONAL
}

\author{
PRE-SERVICE TEACHER TRAINING IN ADAPTED PHYSICAL ACTIVITY: \\ ANALYSIS OF INTERNATIONAL SCIENTIFIC PRODUCTION
}

CAPACITACIÓN PROFESIONAL INICIAL EN ACTIVIDAD FÍSICA ADAPTADA:
ANÁLISIS DE LA PRODUCCIÓN CIENTIIFICA INTERNACIONAL

Patricia Rossi-Andrion*, Carla Ariela Rios Vilaronga**, Mey de Abreu van Munster*

\begin{abstract}
Palavras chave: Atividade Física. Pessoas com deficiência. Capacitação profissional.

Resumo: 0 estudo teve como objetivo analisar a produção científica acerca da formação profissional inicial na área de Atividade Física Adaptada em uma perspectiva internacional. Foi realizada uma revisão sistemática de literatura envolvendo a interface "Atividade Física Adaptada" e "Formação Profissional". Os resultados demonstraram que as pesquisas se concentraram em países do Hemisfério Norte, com maior evidência nos EUA, e que as experiências práticas deveriam ser mais oportunizadas durante a formação inicial com a finalidade de associar teoria com a prática e a infusão do conhecimento; possibilitar o contato direto com as pessoas com deficiência; e influenciar de forma positiva os sentimentos e as atitudes dos estudantes para atuar na AFA. Sendo assim, foi possível concluir a necessidade de se oportunizar mais experiências práticas durante a formação inicial.
\end{abstract}

Keywords:

Physical Activity.

Disabled persons.

Professional training.

Abstract: This study analyzed the scientific production about pre-service teacher training in the area of Adapted Physical Activity from an international perspective. A systematic literature review was carried out involving the interface between 'Adapted Physical Activity' and 'Professional Training'. According to the results, the research was concentrated in countries of the Northern Hemisphere, with higher presence in the USA, and more opportunities for practical experience should be provided during pre-service training in order to associate theory with practice and the introduction of knowledge; to enable direct contact with people with disabilities; and to exert positive influence over students' feelings and attitudes for working in APA. It concludes that more practical experiences must be provided during pre-service training.

Palabras clave: Actividad física. Personas con discapacidad. Capacitación profesional.
Resumen: El estudio tuvo como objetivo analizar la producción científica acerca de la capacitación profesional inicial en el área de Actividad Física Adaptada con una perspectiva internacional. Se realizó una revisión sistemática de literatura bajo la interfaz "Actividad Física Adaptada" y "Capacitación Profesional". Los resultados demostraron que las investigaciones se concentraron en países del Hemisferio Norte, con mayor evidencia en EE.UU. y que las experiencias prácticas deberían tener más oportunidades durante la capacitación inicial, con la finalidad de asociar la teoría con la práctica y de infundir el conocimiento; permitir el contacto directo con las personas con discapacidad; e influir positivamente en los sentimientos y las actitudes de los estudiantes para actuar en la AFA. Así, fue posible concluir la necesidad de dar oportunidades a más experiencias prácticas durante la capacitación inicial.
*Universidade Federal de São Carlos. São Carlos, SP, Brasil.

E-mail: patriciarossi.pr@hotmail.com; munster.mey@gmail.com

**Instituto Federal de São Paulo. São Carlos, SP, Brasil.

E-mail: crios@ifsp.edu.br

Recebido em: 31-03-2019

Aprovado em: 04-06-2019

Publicado em: 27-10-2019

DOI:

https://doi.org/10.22456/1982-8918.91481

(c) (1) (8) Licence 


\section{INTRODUÇÃO}

Atualmente, considera-se que a Atividade Física Adaptada é uma subárea da Educação Física que tem como finalidade o estudo e a intervenção profissional acerca das pessoas com diferentes e peculiares condições para a prática de atividades físicas, desenvolvendo a cultura corporal de movimento (PEDRINELLI; VERENGUER, 2019), podendo ser vista enquanto área de estudo, disciplina de ensino superior e área de atuação em ambientes escolares, esportivos e de reabilitação (CASTRO, 2011).

O termo Atividade Física Adaptada (AFA) começou a ser difundido nos EUA entre as décadas de 1950 e 1970, momento em que se iniciou também a preocupação com a formação profissional, surgindo especializações em AFA nas universidades e o primeiro mestrado em 1968 na State University of New York (SUNY) em Brockport, impulsionando a atividade científica nessa área (CASTRO, 2011).

A partir de então, deu-se início a uma disseminação internacional em outros países, que demonstraram também uma preocupação com a formação acadêmica e profissional envolvendo a AFA. No Brasil, esse marco aconteceu em meados da década de 1980, quando foi identificado que poucos profissionais da Educação Física atuavam na área da Educação Especial, o que alavancou, então, a estruturação de cursos de capacitação na área para iniciar a disseminação de conhecimentos e promover a inserção da disciplina AFA no Ensino Superior (PEDRINELLI; VERENGUER, 2019). Isso culmina com marcos históricos legais, como, por exemplo, a Resolução n. 03/87 do Conselho Federal de Educação, que instituiu a disciplina AFA nos cursos de graduação em Educação Física (PEDRINELLI; VERENGUER, 2019), para atender às demandas da sociedade.

Desde então, pesquisas envolvendo a formação profissional e a AFA vêm sendo desenvolvidas com a finalidade de compreender como acontece tal ação, quais os avanços, limitações e de que maneira interfere na atuação do profissional (LOUZADA, 2016; MAHL, 2016).

A formação profissional tem como finalidade envolver uma formação básica sólida, que propicie o conhecimento dos fundamentos científicos e sociais de suas competências de trabalho, que associe teoria e prática por meio de estágios supervisionados e capacitação em serviço e que oportunize o aproveitamento da formação e de experiências anteriores advindas de instituições de ensino e de outras atividades (CASTRO; AMORIM, 2015).

A formação profissional tem duas vertentes, a inicial e a continuada. A formação inicial refere-se ao ensino superior, caracterizado como o ponto de partida para o ingresso no mercado de trabalho, habilitando sua atuação em determinada área (MIZUKAMI, 2002). Já a formação continuada envolve demais cursos dos quais os profissionais participam após a formação inicial, sendo compreendida como um processo permanente de aperfeiçoamento e atualização das experiências adquiridas para ampliar suas competências profissionais como complemento/capacitação (SACRISTÁN, 2000; MIZUKAMI, 2002; CASTRO; AMORIM, 2015).

Chicon, Peterle e Santana (2014), em uma pesquisa bibliográfica realizada com artigos nacionais entre os anos de 2000 e 2010, encontraram cinco trabalhos publicados sobre a formação em AFA, sendo dois sobre a formação inicial e três sobre a continuada. 
No que se refere à formação inicial, verificou-se o despreparo ao lidar com as pessoas com deficiência, decorrente da falta de experiências, precariedade do estágio supervisionado e falta de interdisciplinaridade nos cursos superiores de formação inicial, causando insegurança e dificuldades para a atuação profissional, tanto por recém-formados como por professores de carreira. Mesmo com a disciplina AFA incluída nos currículos dos cursos de graduação, tal pesquisa aponta que outras lacunas ocorrem no processo formativo/ profissional sendo necessária uma formação posterior por parte dos profissionais. Quanto à formação continuada, tanto os professores que tiveram como os que não tiveram uma formação profissional voltada para a perspectiva inclusiva relataram insegurança diante das diferenças no cotidiano escolar, buscando qualificar-se por meio de cursos, leituras e trocas de experiências, a fim de aumentar seu conhecimento e ressignificar a sua prática.

Ainda quanto à formação inicial, Rossi e Munster (2013) também apontam que mesmo o curso superior apresentando condições estruturais e curriculares para atender às demandas da inclusão, ainda há poucas disciplinas que realizam a interface/interdisciplinaridade com a Educação Física Adaptada (EFA), justificando o fato da carga horária da disciplina isolada ser insuficiente para o que se espera de um profissional recém-formado. As autoras defendem a importância da interdisciplinaridade entre a EFA e as demais disciplinas, maior articulação entre as atividades de ensino, extensão e pesquisa, e maior aproximação com as disciplinas de estágio curricular.

Silva e Drigo (2012), Louzada (2016) e Oliveira (2017) realizaram pesquisas voltadas aos currículos das universidades de ensino superior do Brasil. Esses estudos, de modo geral, verificaram que não há padrões nas instituições de como deve ser desenvolvida a formação inicial dos profissionais envolvendo a AFA para torná-los mais preparados para atuação. Isso leva a uma limitação nas pesquisas que tentam identificar de que forma a disciplina AFA tem sido abordada na Educação Superior, pois não há um delineamento de como são conduzidas, existindo um distanciamento entre a formação inicial para contextos de diversidade e inclusão, a realidade escolar e o desatrelamento entre teoria e prática.

Mahl (2016) conclui que a oferta apenas da AFA nos cursos de formação inicial e continuada não garante a preparação dos profissionais para a atuação envolvendo a perspectiva inclusiva, mas é fundamental para permitir o desenvolvimento de novas culturas, políticas e práticas de inclusão.

A formação profissional inicial é um tema recorrente nas pesquisas envolvendo a Atividade Física Adaptada no Brasil, pois é a partir dela que serão proporcionados os conhecimentos dos fundamentos científicos que envolvem a futura atuação na área em questão. Sendo assim, torna-se fundamental sua compreensão, como vem ocorrendo internacionalmente, a fim de encontrar lacunas e novos caminhos a serem percorridos. Visando, então, respaldar as investigações e políticas públicas nacionais voltadas à formação profissional inicial na área de AFA, é necessário vislumbrar como esse cenário tem se estabelecido nos demais países.

Com isso, este estudo tem como objetivo analisar a produção científica acerca da formação profissional inicial na área de Atividade Física Adaptada em uma perspectiva internacional. 


\section{MÉTODO}

Esta pesquisa foi baseada em uma revisão sistemática de literatura, que permite aumentar o potencial de uma busca, a fim de encontrar o maior número possivel de resultados de uma maneira organizada, sendo constituída em um trabalho reflexivo, crítico e compreensivo a respeito do material analisado (COSTA; ZALTOWSKI, 2014).

A presente revisão foi desenvolvida com base nos critérios propostos pelo PRISMA Preferred Reporting Items For Systematic Reviews and Meta-analyses (MOHER et al., 2009), grupo que propõe um conjunto mínimo de itens baseados em evidências que devem ser considerados em estudos de revisão sistemática e meta-análise com a finalidade de aumentar a confiabilidade dos dados coletados.

Para o levantamento dos dados foram considerados os seguintes critérios:

a) Tipo: artigos científicos publicados na íntegra em periódicos revisados por pares;

b) Tema: formação profissional e atividade física adaptada;

c) Período: sem determinação;

d) Idioma: inglês;

e) Base de dados: SportDiscus, Bireme, PubMed, SciELO, por meio do Portal de Periódicos CAPES/MEC;

f) Palavras-chave (descritores) utilizados: (adapted physical education OR adapted physical activity OR inclusive physical education) AND (professional training OR pre-service training OR teacher training OR vocational training OR teacher educat ${ }^{\star}$ ) nos itens título, autor ou assunto.

Os descritores utilizados foram embasados no DeCS (Descritores em Ciências da Saúde) e nas palavras frequentes encontradas em estudos sobre o tema. Para aumentar a fidedignidade desse levantamento, o objetivo do estudo e os descritores foram encaminhados a três juízes graduados na área da Educação Física com especialidade na área da Atividade Física Adaptada para verificar a concordância.

As buscas foram realizadas durante o mês de novembro de 2018. Os artigos encontrados na pesquisa foram selecionados através da leitura de seus títulos e resumos e foram excluídos:

a) ocorrências repetidas;

b) publicações que não estavam disponíveis na íntegra;

c) artigos de revisão sistemática e de estudos teóricos;

d) artigos que não se relacionavam com o propósito aqui presente (formação profissional que não envolvesse a Atividade Física Adaptada);

e) artigos sobre formação continuada e em serviço;

Após a leitura de todos os artigos elegíveis para inclusão na revisão foi obtido o resultado do número final de artigos para a análise criteriosa. A Imagem 1 apresenta um fluxograma referente às etapas da revisão sistemática. 
Imagem 1- Fluxograma das estapas da revisão sistemática.

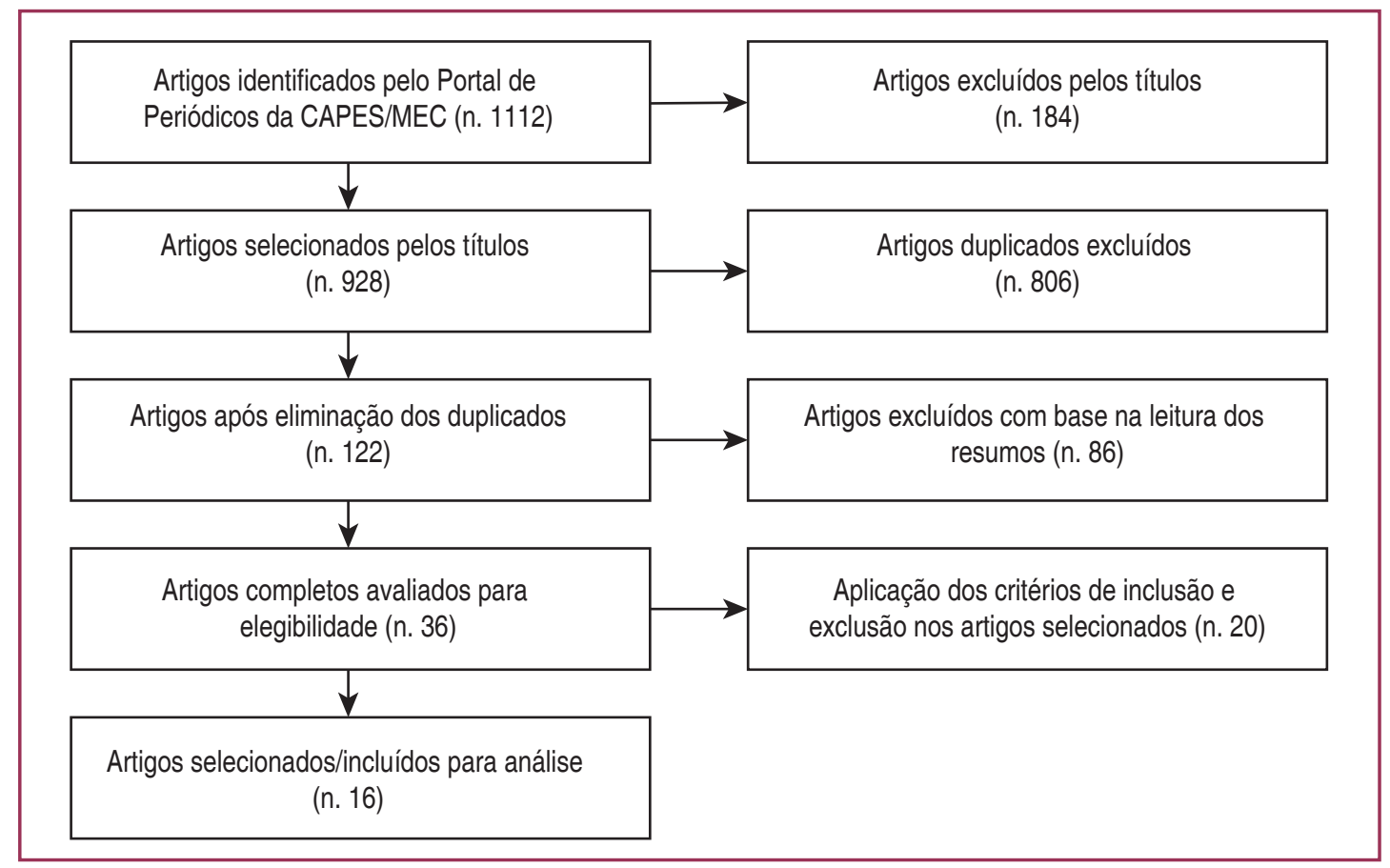

Fonte: Desenvolvido pelos autores, baseado no modelo do Grupo PRISMA - Preferred Reporting Items For Systematic Reviews and Meta-analyses (MOHER et al., 2009).

Os estudos selecionados foram lidos e catalogados para análise temática, que, segundo Braun e Clarke (2006), é um método que identifica, analisa e relata padrões/temas, organizando e defendendo um conjunto de dados com detalhes. Os autores descrevem seis etapas: 1. Familiariazação com os dados por meio de anotações; 2. Geração de códigos iniciais embasados nessas anotações, citações e destaques realizados pelo pesquisador; 3. Pesquisa por temas, verificando a recorrência dos dados e sua relevância; 4. Revisão dos temas selecionados, estabelecenco relações entre eles; 5 . Definir e nomear os temas; 6 . Escrita sobre os temas, contextualizando a literatura existente.

Sendo assim, após a leitura dos artigos na íntegra, foram dispostos em uma tabela o título, autores, revista, ano e país de publicação, objetivos, método, principais resultados e considerações finais/conclusões. Com relação aos resultados e considerações finais/ conclusões, foram realizadas anotações e destaques acerca da formação inicial em AFA. A partir dessas anatoções, foi realizada uma leitura mais cuidadosa e verificou-se a recorrência de temas (como, por exemplo, a associação da teoria e prática durante as aulas da graduação, a infusão do conhecimento a ser desenvolvido nas disciplinas do curso, os sentimentos dos alunos ao se trabalhar com a AFA, as atitudes relacionadas às experiências vividas na formação inicial, o fato de se sentirem mais bem preparados a partir do contato direto com as pessoas com deficiência por meio dos estágios), mostrando sua relevância para a apresentação dos resultados aqui presentes. Esses temas foram selecionados, relacionados e dispostos em três grandes categorias: I) Associação da teoria com a prática e a infusão do conhecimento; II) Contato direto com as pessoas com deficiência; e III) Melhoria dos sentimentos e das atitudes ao atuar com pessoas com deficiência. Essa divisão permitiu que fossem realizadas as discussões e contextualizações com literatura, como apresenta a seguir, no item 3. 


\section{RESULTADOS E DISCUSSÃO}

A partir do levantamento realizado por meio do Portal de Periódicos da Capes, foram selecionados 16 artigos escritos em língua inglesa publicados no período entre 2002 e 2018. Os estudos foram publicados em seis diferentes revistas acadêmico-científicas: Adapted Physical Activity Quarterly $(\mathrm{n}=3)$; Physical Education and Sport Pedagogy $(\mathrm{n}=3)$; European Physical Education Review ( $n=3)$; The Physical Educator $(n=2)$; Palaestra $(n=2)$; Clinical $(n=1)$; Acta Gymnica $(n=1)$ e Springer Science $(n=1)$.

Verificou-se que as pesquisas foram desenvolvidas em diferentes países, como: EUA $(n=9)$; Reino Unido $(n=2)$; Canadá $(n=1)$; Itália $(n=1)$; Irlanda $(n=1)$; Turquia $(n=1)$ e Espanha $(n=1)$. Tais dados sugerem a preocupação com que a formação inicial em Atividade Física Adaptada foi disseminada por vários países pertencentes à América do Norte e à Europa ao longo das últimas duas décadas. Todavia, a concentração de pesquisas envolvendo essa temática permanece nos EUA, país precursor na formação superior em AFA e onde datam as primeiras pesquisas na área desde 2002, demonstrando preocupação com a competência profissional e a necessidade de comunicação por quem produz o conhecimento expresso pela literatura (CASTRO, 2011).

A seguir o Quadro 1 apresenta os estudos encontrados, os autores e o país em que foram desenvolvidos, bem como seus objetivos.

Quadro 1 - Relação dos estudos incluídos na revisão de literatura.

\begin{tabular}{|c|c|c|c|}
\hline Autor (ano) & Pais & Título/Revista & Objetivo \\
\hline $\begin{array}{l}\text { HODGE; } \\
\text { TANNEHILL; } \\
\text { KLUGE (2002) }\end{array}$ & EUA & $\begin{array}{l}\text { Exploring the meaning of } \\
\text { practicum experiences for PETE } \\
\text { students. Adapted Physical } \\
\text { Activity Quartely (APAQ). }\end{array}$ & $\begin{array}{l}\text { Explorar o significado da experiência prática } \\
\text { dos estudantes da educação de professores } \\
\text { em EF. }\end{array}$ \\
\hline $\begin{array}{l}\text { HODGE et al. } \\
(2002)\end{array}$ & EUA & $\begin{array}{l}\text { Comparison of practicum } \\
\text { types in changing preservice } \\
\text { teachers' attitudes and perceived } \\
\text { competence. Adapted Physical } \\
\text { Activity Quartely (APAQ). }\end{array}$ & $\begin{array}{l}\text { Comparar o efeito de dois tipos de prática } \\
\text { (fora e dentro do campo) acerca das atitudes } \\
\text { dos estudantes do curso de educação } \\
\text { de professores em EF em relação aos } \\
\text { alunos com deficiência física ou deficiência } \\
\text { intelectual severa/moderada. }\end{array}$ \\
\hline $\begin{array}{l}\text { DUCHANE et } \\
\text { al. (2008) }\end{array}$ & EUA & $\begin{array}{l}\text { Pre-service Physical Educator } \\
\text { attitude toward teaching } \\
\text { students with disabilities. Clinical } \\
\text { Kinesiology. } \\
\end{array}$ & $\begin{array}{l}\text { Examinar as atitudes dos graduandos em EF } \\
\text { em relação às pessoas com deficiência. }\end{array}$ \\
\hline $\begin{array}{l}\text { VICKERMAN; } \\
\text { COATES (2009) }\end{array}$ & $\begin{array}{l}\text { Reino } \\
\text { Unido }\end{array}$ & $\begin{array}{l}\text { Trainee and recently qualified } \\
\text { physical education teachers' } \\
\text { perspectives on including children } \\
\text { with special educational needs. } \\
\text { Physical Education and Sport } \\
\text { Pedagogy. }\end{array}$ & $\begin{array}{l}\text { Analisar as visões e opiniões do estagiário } \\
\text { e dos professores recém-formados em EF } \\
\text { para obter uma visão sobre suas opiniões e } \\
\text { experiências de formação inicial para incluir } \\
\text { crianças com Necessidades Educacionais } \\
\text { Especiais (NEE). }\end{array}$ \\
\hline $\begin{array}{l}\text { RUST; } \\
\text { SINELNIKOV } \\
(2010)\end{array}$ & EUA & $\begin{array}{l}\text { Practicum in a Self-contained } \\
\text { Enviroment: pre-service teacher } \\
\text { perceptions of teaching students } \\
\text { with disabilities. The Physical } \\
\text { Educator. }\end{array}$ & $\begin{array}{l}\text { Explorar a percepção de um professor } \\
\text { recém-formado em relação aos alunos } \\
\text { com deficiência e a preparação profissional } \\
\text { em um ambiente independente durante } 0 \\
\text { estágio. }\end{array}$ \\
\hline
\end{tabular}




\begin{tabular}{|c|c|c|c|}
\hline COATES (2012) & $\begin{array}{l}\text { Reino } \\
\text { Unido }\end{array}$ & $\begin{array}{l}\text { Teaching inclusively: are } \\
\text { secondary physical education } \\
\text { student teachers sufficiently } \\
\text { prepared to teach in inclusive } \\
\text { environments? Physical } \\
\text { Education and Sport Pedagogy. }\end{array}$ & $\begin{array}{l}\text { Determinar se os professores de EF estão } \\
\text { suficientemente preparados para ensinar } \\
\text { as crianças com NEE, examinando sua } \\
\text { formação inicial. }\end{array}$ \\
\hline JEONG (2013) & EUA & $\begin{array}{l}\text { Pre-service Physical Educator } \\
\text { attributes and confidence: } \\
\text { in teaching individuals with } \\
\text { disabilities. Palaestra. }\end{array}$ & $\begin{array}{l}\text { Examinar os atributos e confiança dos } \\
\text { graduandos em EF em relação à prática da } \\
\text { disciplina AFA. }\end{array}$ \\
\hline $\begin{array}{l}\text { WILKINSON et } \\
\text { al. (2013) }\end{array}$ & Canadá & $\begin{array}{l}\text { Student teacher experiences } \\
\text { in a service-learning project for } \\
\text { children with attention-deficit } \\
\text { hyperactivity disorder. Physical } \\
\text { Education and Sport Pedagogy. }\end{array}$ & $\begin{array}{l}\text { Explorar as experiências dos alunos da } \\
\text { formação de professores em EF em um } \\
\text { projeto do estágio voluntário para crianças } \\
\text { com Transtorno do Déficit de Atenção e } \\
\text { Hiperatividade. }\end{array}$ \\
\hline $\begin{array}{l}\text { NARDO et al. } \\
(2014)\end{array}$ & Itália & $\begin{array}{l}\text { Attitudes of preservice physical } \\
\text { educators toward individuals } \\
\text { with disabilities at University } \\
\text { of Parthenope of Napoli. Acta } \\
\text { Gymnica. }\end{array}$ & $\begin{array}{l}\text { Examinar os efeitos de um curso de } \\
\text { graduação em AFA sobre as atitudes dos } \\
\text { graduandos acerca dos indivíduos com } \\
\text { deficiências. }\end{array}$ \\
\hline $\begin{array}{l}\text { TINDALL et al. } \\
(2014)\end{array}$ & Irlanda & $\begin{array}{l}\text { Pre-service teachers' attitudes } \\
\text { towards children with disabilities: } \\
\text { an Irish perspective. European } \\
\text { Physical Education Review. }\end{array}$ & $\begin{array}{l}\text { Examinar o impacto de um programa de } \\
\text { AFA de dez semanas sobre as atitudes e } \\
\text { percepções de } 64 \text { professores irlandeses } \\
\text { em formação inicial em relação ao ensino de } \\
\text { crianças e jovens com deficiências física e } \\
\text { intelectual. }\end{array}$ \\
\hline $\begin{array}{l}\text { WOODTRUFF; } \\
\text { SINELNIKOV, } \\
2014\end{array}$ & EUA & $\begin{array}{l}\text { Teaching young adults with } \\
\text { disabilities through service } \\
\text { learning. European Physical } \\
\text { Education Review. }\end{array}$ & $\begin{array}{l}\text { Examinar o que os alunos que aprendem } \\
\text { a ensinar jovens adultos com deficiência } \\
\text { consideram significativo para a evolução } \\
\text { desses alunos durante uma experiência de } \\
\text { campo. }\end{array}$ \\
\hline $\begin{array}{l}\text { GOMEZ; } \\
\text { BARTOLL; } \\
\text { PUIG (2015) }\end{array}$ & Espanha & \begin{tabular}{|l|} 
The impact of service learning \\
on the training of pre-service \\
teachers: Analysis from a physical \\
education subject. European \\
Physical Education Review.
\end{tabular} & $\begin{array}{l}\text { Discutir os efeitos do uso da aprendizagem } \\
\text { de serviço em professores durante a } \\
\text { formação inicial. }\end{array}$ \\
\hline $\begin{array}{l}\text { TALIAFERRO; } \\
\text { HAMMOND; } \\
\text { WYANT (2015) }\end{array}$ & EUA & $\begin{array}{l}\text { Preservice Physical Educators' } \\
\text { Self-Efficacy Beliefs Toward } \\
\text { Inclusion: The Impact of } \\
\text { Coursework and Practicum. } \\
\text { Adapted Physical Activity } \\
\text { Quartely (APAQ). }\end{array}$ & $\begin{array}{l}\text { Investigar o efeito da conclusão do curso de } \\
\text { AFA com associação da prática em campo } \\
\text { na formação inicial de educadores físicos no } \\
\text { que se refere a sua autoeficácia em relação } \\
\text { à inclusão de indivíduos com deficiências. }\end{array}$ \\
\hline KWON (2018) & EUA & $\begin{array}{l}\text { Status of introductory APE course } \\
\text { and infusion in PETE program. } \\
\text { Palaestra. }\end{array}$ & $\begin{array}{l}\text { Descrever o atual status do curso introdutório } \\
\text { de AFA nos cursos de formação de } \\
\text { professores em Educação Física e examinar } \\
\text { como os conceitos de deficiência são } \\
\text { difundidos no currículo. }\end{array}$ \\
\hline $\begin{array}{l}\text { LAYNE; } \\
\text { BLASINGAME } \\
\text { (2018) }\end{array}$ & EUA & $\begin{array}{l}\text { Analysis of a Physical Education } \\
\text { Teacher Education Field } \\
\text { Experience of Working One-on- } \\
\text { One With Students With Severe } \\
\text { and Profound Disabilities in a } \\
\text { Self-Contained Environment. The } \\
\text { Physical Educator. }\end{array}$ & $\begin{array}{l}\text { Analisar as percepções de professores e } \\
\text { alunos da formação de professores em } \\
\text { Educação Física acerca de uma experiência } \\
\text { de campo projetada para fornecer aos } \\
\text { estudantes oportunidade de trabalhar com } \\
\text { um aluno com deficiências profundas. }\end{array}$ \\
\hline
\end{tabular}




\begin{tabular}{|c|c|c|c|}
\hline $\begin{array}{l}\text { OZNACAR; } \\
\text { ERDAG (2018) }\end{array}$ & Turquia & $\begin{array}{l}\text { Physical education and sports } \\
\text { education candidate } \\
\text { students' awareness and } \\
\text { knowledge status about physical } \\
\text { education lessons designed for } \\
\text { physically disabled } \\
\text { individuals. Springer Science. }\end{array}$ & $\begin{array}{l}\text { Investigar a conscientização e conhecimento } \\
\text { dos alunos do curso de Educação Física } \\
\text { sobre as aulas de Educação Física para } \\
\text { pessoas com deficiência. }\end{array}$ \\
\hline
\end{tabular}

Fonte: próprios autores.

Dos artigos encontrados, verificou-se uma predominância de pesquisas com abordagem qualitativa $(n=12)$, sendo os instrumentos de coleta de dados entrevistas, observações e reflexões escritas dos participantes (graduandos e professores recém-formados), evidenciando a percepção destes no que se referia a sua formação profissional inicial acerca da AFA para pessoas com deficiência. Apenas quatro estudos utilizaram uma abordagem quantitativa, em que foram aplicadas escalas avaliativas de atitudes em graduandos de Educação Física.

Os resultados apontaram que quatorze estudos $(87,5 \%)$ abordaram a percepção dos estudantes que estavam em formação inicial envolvendo a AFA, evidenciando a importância de dar a voz a quem tem o papel principal em atuar diante da inclusão nas escolas com pessoas com deficiência, com a finalidade de compreender se eles consideravam estar preparados ou não para tal situação.

A temática "experiência prática" foi citada nos resultados de todos os artigos e considerada de extrema importância na preparação dos estudantes em formação inicial para se sentirem confiantes ao atuar junto aos alunos com deficiência. Essa experiência prática envolveu atividades em que os participantes acompanhavam e vivenciavam de perto o trabalho realizado com as pessoas com deficiência, como, por exemplo, estágios supervisionados dentro e fora do campus universitário, estágios curriculares em escolas de Educação Especial e Regular; projetos de extensão e projetos comunitários que envolviam as pessoas com deficiência.

Para facilitar a discussão dos dados, foram identificadas três categorias temáticas que abordaram as vertentes da experiência prática, sendo elas: 1) Associação da teoria com a prática e a infusão do conhecimento; 2) Contato direto com as pessoas com deficiência; 3) Melhoria dos sentimentos e das atitudes ao atuar na AFA.

\subsection{ASSOCIAÇÃO DA TEORIA COM A PRÁTICA E A INFUSÃO DO CONHECIMENTO}

A associação da teoria com a prática durante os cursos de formação inicial foi uma temática relevante nos artigos encontrados no presente estudo. De acordo com Talliaferro, Hammond e Wyant (2015), Gomez, Bartoll e Puig (2015) e Oznacare Erdag (2018), os resultados dos seus estudos demonstraram que deveria ocorrer a combinação teórica da AFA junto às experiências práticas, fazendo com que a teoria acompanhasse a prática com a finalidade de melhorar a competência de ensino e 0 aumento da compreensão cultural da deficiência, além do aumento do conhecimento e da conscientização sobre a inclusão. Foi citada, também, a importância em diversificar para não trabalhar com os conteúdos tradicionais (COATES, 2012).

Layne e Blasingame (2018) afirmaram com sua pesquisa que o tempo despendido nas atividades práticas durante a formação inicial deveria ser maior, pois há muita teoria e pouca 
prática. Segundo as autoras, essa experiência prática deveria ser estendida por mais tempo e direcionada para atividades em campo, para que houvesse o aumento de tempo de instrução para proporcionar a melhoria da confiança e o entusiasmo nos estudantes dos programas de formação inicial em AFA, e assim, consequentemente, melhorar a instrução ao se trabalhar com alunos com deficiência.

Segundo Hodge Tannehill e Kluge (2002), Vickerman e Coates (2009), Jeong (2013), Wilkinson et al. (2013), Nardo et al. (2014) e Tindall et al. (2014), seria de extrema importância que as experiências práticas em ambiente real, como, por exemplo, em escolas de ensino regular que atendem alunos com deficiência ou em escolas de Ensino Especial, fossem incorporadas e oportunizadas durante a graduação, para que pudesse oferecer aos estudantes em formação inicial um treinamento adequado, proporcionando mais conhecimento e maior preparo acerca da inclusão, com um consequente aumento na qualidade de ensino para todos.

Juntamente com a teoria e a prática, verificou-se a importância de oferecer aos estudantes a infusão do conhecimento entre as disciplinas ao longo dos cursos de formação inicial. Kwon (2018), em um estudo empírico do qual participaram professores de 75 faculdades representando 31 estados dos EUA por meio de uma pesquisa online, descreveu 0 atual status do curso introdutório de AFA nos cursos de formação de professores em Educação Física e examinou como os conceitos de deficiência estavam sendo difundidos no currículo. Os resultados apontaram pouca oferta de disciplinas que envolviam a AFA e a escassez de experiências práticas, defendendo a importância da infusão do conhecimento sobre deficiência em outras disciplinas, pois essa infusão poderia favorecer discussões, simulações, equipamentos, modificações e estratégias de ensino e de conhecimento.

Isso corrobora o estudo de Rossi e Munster (2013) no Brasil, que relatou a importância de propor a interface do conhecimento AFA entre as disciplinas da graduação para um melhor aproveitamento acadêmico, com uma consequente melhora na preparação para atuar com as pessoas com deficiência.

\subsection{CONTATO DIRETO COM AS PESSOAS COM DEFICIÊNCIA}

Essa vertente relacionou a importância da experiência prática com o contato direto com as pessoas com deficiência, podendo este ser por meio de estágios acadêmicos e/ou de experiências pessoais anteriores ao início da formação inicial, como, por exemplo, ter familiares e/ou amigos com deficiência. Afinal, a formação profissional, além de envolver uma formação básica sólida, que associe teoria e prática por meio de estágios supervisionados, ainda envolve as experiências anteriores advindas de instituições de ensino e de outras atividades (CASTRO; AMORIM, 2015).

Segundo Duchane et al. (2018), seu estudo concluiu que quanto mais experiência e contato direto com as pessoas com deficiência o formando tivesse, melhor seria seu sentimento em relação à atuação frente à inclusão.

Tindall et al. (2014) e Woodruff e Sinelnikov (2014) demonstraram em seus estudos que, quando há experiências práticas em estágios fora do campus (em ambiente real), há, consequentemente, um aumento da percepção sobre a inclusão, que pode gerar mudanças de discurso e do preconceito acerca das pessoas com deficiência e, também, da compreensão 
cultural da deficiência com a finalidade de olhar 0 aluno acerca das suas funcionalidades. Concomitantemente, a experiência prática pode proporcionar uma maior apreciação pela vida e a compreensão das necessidades dos alunos com deficiência, trazendo benefícios para a formação profissional (LAYNE; BLASINGAME, 2018; GOMEZ; BARTOLL; PUIG, 2015).

\subsection{MELHORIA DOS SENTIMENTOS E DAS ATITUDES AO ATUAR COM PESSOAS COM DEFICIÊNCIA}

Além do contato direto, segundo Woodruff e Sinelnikov (2014), a experiência prática pode influenciar de forma positiva nas seguintes atitudes:

a) Mudança de discurso ao tratar-se sobre inclusão, buscando a equidade durante as aulas;

b) Diminuição do preconceito, deixando a deficiência de lado e buscando a funcionalidade para ao ir ao encontro das necessidades de cada aluno;

c) Desenvolvimento de relacionamentos e comunicação adequados ao trabalhar com o público em questão.

Hodge et al. (2002), Duchane et al. (2008) e Nardo et al. (2014) verificaram que, quando houve experiência prática prévia sobre a deficiência, melhor preparo acadêmico e contato com mais conteúdos envolvendo especificamente a Atividade Física Adaptada durante os cursos de formação inicial, mais bem preparados academicamente os formandos se sentiam e maior foi o resultado da atitude positiva e da intenção de inclusão por parte deles. Os resultados desses estudos também demonstraram que a experiência prática estava relacionada com o aumento da confiança em proporcionar a inclusão, com a melhoria da comunicação junto aos alunos com deficiência e demais profissionais para um trabalho multidisciplinar e com a diminuição de incertezas ao se trabalhar com esse público.

Vickerman e Coates (2009), Woodruff e Sinelnikov (2014), Tindall et al. (2014) e Gomez, Bartoll e Puig (2015) verificaram em seus estudos que o aumento da atitude positiva e da confiança estava relacionado aos estágios fora do campus e em escolas de Educação especial ou escola regular, que proporcionaram aos estudantes de formação inicial um contato maior com as pessoas com deficiência em ambiente real, influenciando diretamente na diminuição da ansiedade, no aumento da percepção em relação à inclusão e na diminuição das incertezas futuras. Além do aumento da confiança vinculado ao maior contato nos estágios, Woodruff e Sinelnikov (2014) e Talliaferro, Hammond e Wyant (2015) verificaram que esse ambiente ainda possibilitou feedback de diferentes profissionais (como professores de sala de aula regular, diretores e assistentes) e o desenvolvimento positivo de relacionamento e comunicação dos formandos em relação aos alunos com deficiência.

Além das atitudes, a experiência prática pode influenciar positivamente nos sentimentos e emoções dos estudantes em formação inicial ao se trabalhar com alunos com deficiência. Segundo Woodruff e Sinelnikov (2014), os estudantes passaram a se sentir mais bem preparados para enfrentar as diferentes necessidades desses indivíduos na sua futura atuação. Tais sentimentos foram desenvolvidos com base em uma formação inicial que possibilitou as experiências práticas em ambientes reais, fora dos campi, diretamente com alunos com deficiência e com contato com profissionais que disponibilizaram suas experiências em serviço (HODGE et al., 2002; DUCHANE et al. 2008; NARDO et al. 2014) 
A compilação desses resultados demonstrou que a experiência prática é a principal responsável por relacionar várias vertentes para uma melhor formação inicial, pois são essas experiências práticas por meio de estágios em ambientes reais (que deveriam ser mais oportunizados), juntamente com a teoria, que interferem positivamente nas atitudes e nos sentimentos dos estudantes, tornando-os mais bem preparados para a atuação frente à inclusão de pessoas com deficiência.

Esses apontamentos demonstram a importância de os programas de formação inicial oferecerem conteúdos e experiências mais eficazes, principalmente no que se refere aos estágios, proporcionando uma melhor qualidade de ensino para todos e, consequentemente, aumentando a atitude positiva em relação às pessoas com deficiência e a intenção de inclusão (NARDO et al. 2014), juntamente com aumento dos sentimentos de confiança.

De modo geral, os resultados apresentados corroboram aqueles encontrados no Brasil, que demonstram ocorrer a falta de experiências práticas, a precariedade do estágio supervisionado e a falta de interdisciplinaridade nos cursos superiores de formação inicial, que podem proporcionar insegurança e dificuldades para a atuação profissional (CHICON, PETERLE, SANTANA, 2014).

\section{CONSIDERAÇÕES FINAIS}

Este estudo apresentou o que vem sendo pesquisado ao longo das últimas décadas no âmbito internacional envolvendo a formação profissional em Educação Física Adaptada.

Verificou-se que a concentração de pesquisas envolvendo essa temática permanece nos EUA, país precursor na formação superior em AFA. Apesar dos vários estudos desenvolvidos no Brasil acerca da temática em questão, esses dados não foram divulgados em periódicos internacionais, e, portanto, ficam circunscritos às publicações nacionais e em português. Isso pode estar relacionado com a falta de políticas científicas com fomento para tradução e custeio de submissões enfrentadas na área acadêmica no Brasil.

De modo geral, os estudos demonstraram que os estudantes não se sentiam preparados para trabalhar com as pessoas com deficiência, sendo ressaltado, principalmente, o pouco contato com esse público ao longo dos cursos de formação.

Assim, verificou-se a importância em oportunizar mais experiências práticas envolvendo a AFA durante o processo de formação inicial, pois é a partir dela que há o contato direto com as pessoas com deficiência em ambiente real, influenciando positivamente em suas atitudes e sentimentos frente a atuação na AFA e ao processo de inclusão. Além disso, foi verificada a importância em se aprofundar mais os conteúdos da AFA durante os cursos de formação, sugerindo a infusão do conhecimento e não disciplinas isoladas com pouca intervenção prática.

Sendo assim, pode-se inferir que as angústias, anseios e dificuldades na formação inicial no que se refere à Atividade Física Adaptada acontecem de forma generalizada nos cursos de formação superior tanto no âmbito nacional quanto internacional. 


\section{REFERÊNCIAS}

BRAUN, Virginia; CLARKE, Victoria. Using thematic analysis in psychology. Qualitative Research in Psychology, v. 3, n. 2, p. 77-101, 2006.

CASTRO, Eliane M. A disciplina atividade física adaptada. In: CASTRO, Eliane M. Atividade física adaptada. Ribeirão Preto: Tecmedd, 2011. p. 27-37.

CASTRO; Marcelo M. C.; AMORIM, Rejane M. A. A formação inicial e a continuada: diferenças conceituais que legitimam um espaço permanente de vida. Caderno Cedes, v. 35, n. 95, p. $37-$ 55, abr. 2015.

CHICON, José F.; PETERLE, Ludmila. L.; SANTANA, Monique A. G. Formação, Educação Física e Inclusão: um estudo em periódicos. Revista Brasileira de Ciências do Esporte v. 36 , n. 2, supl., p. S830-S845, abr./jun. 2014.

COATES, Janine K. Teaching inclusively: are secondary physical education student teachers sufficiently prepared to teach in inclusive environments? Physical Education and Sport Pedagogy, v. 17, n. 4, p. 349-365, 2012.

COSTA, Angelo B.; ZALTOWSKI, Ana Paula C. Como escrever um artigo de revisão sistemática. In: KOLLER, Silvia H.; COUTO, Maria C. P. P.; HOHENDORFF, Jean V. (org.). Métodos de pesquisa: manual de produção científica. Porto Alegre: Penso, 2014. p. 55-70.

DUCHANE, Kim A. et al. Pre-service Physical Educator attitude toward teaching students with disabilities. Clinical Kinesiology, v. 3, n. 62, p. 16-20, 2008.

GOMEZ, Jesus G.; BARTOLL, Oscar C.; PUIG, Manuel M. The impact of service learning on the training of pre-service teachers: Analysis from a physical education subject. European Physical Education Review, v. 21, n. 4, p. 467-484, 2015.

HODGE, Samuel R.; TANNEHILL, Deborah.; KLUGE, M. A. Exploring the meaning of practicum experiences for the PETE students. Adapted Physical Activity Quarterly, v. 20, p. 381-399, 2002.

HODGE, Samuel R. et al. Comparison of practicum types in changing preservice teachers' attitudes and perceived competence. Adapted Physical Activity Quarterly, v. 19, p. 155-171, 2002.

JEONG, Mihye. Pre-service Physical Educator attributes and confidence: in teaching individuals with disabilities. Palaestra, v. 27, n. 4, p. 11-13, 2013.

KWON, Eun $\mathrm{H}$. Status of introductory APE course and infusion in PETE program. Palaestra, v. 32, n. 1, p. 32-39, 2018.

LAYNE, Todd E.; BLASINGAME, Jennifer. Analysis of a Physical Education Teacher Education field experience of working one-on-one with students with severe and profound. The Physical Educator, v. 75, p. 683-700, 2018.

LOUZADA, Juliana C. A. Inclusão educacional: em foco a formação de professores de Educação Física. 2016. 127f. Dissertação (Mestrado em Educação) - Faculdade de Filosofia e Ciência, Universidade Estadual Paulista, Marília, 2016. 
MAHL, Eliane. Programa de formação continuada para professores de Educação Física: possibilidades para a construção de saberes sobre a inclusão de alunos com deficiência. 2016. 268f. Tese (Doutorado em Educação Especial) - Programa de Pós-graduação em Educação Especial, Universidade Federal de São Carlos, São Carlos, 2016.

MIZUKAMI, Maria G. N. Formação de professores, conhecimento da docência e casos de ensino. In: REALI, Aline M. M. R.; MIZUKAMI, Maria G. N. (orgs). Formação de professores: práticas pedagógicas e escola. São Carlos: EdUFSCar, 2002. 151-174.

MOHER, David et al. Preferred reporting items for systematic reviews and meta-analyses: the PRISMA statement. Annals of Internal Medicine, PubMed, v. 151, p. 264-269, 2009.

NARDO, Marco et al. Attitudes of preservice physical educators toward individuals with disabilities at University Parthenope of Napoli. Acta Gymnica, v. 44, n. 4, p. 211-221, 2014.

OLIVEIRA, Ana Carolina S. Formação continuada na perspectiva colaborativa: subsídios para inclusão nas aulas de Educação Física. 2017. 200f. Tese (Doutorado em Educação Especial) - Programa de Pós-graduação em Educação Especial, Universidade Federal de São Carlos, São Carlos, 2017.

OZNACAR, Behcet; ERDAG, Deniz. Physical education and sports education candidate students' awareness and knowledge status about physical education lessons designed for physically disabled individuals. Springer Science, Online, v.1, 2018. Disponível em: https://link. springer.com/article/10.1007\%2Fs11135-018-0716-7 Acesso em: 20 out. 2018.

PEDRINELLI, Verena J.; VERENGUER, Rita C. G. Educação física adaptada: introdução ao universo de possibilidades. In: GREGUOL, Marcia; COSTA, Roberto F. (org.). Atividade física adaptada: qualidade de vida para pessoas com necessidades especiais. 2. ed. Barueri: Manole, 2019. p. 1-27.

ROSSI, Patrícia; MUNSTER, Mey de A. Formação profissional em Educação Física Adaptada: um estudo de caso. In: ENCONTRO DA ASSOCIAÇÃO BRASILEIRA DE PESQUISADORES EM EDUCAÇÃO ESPECIAL, 8. [Anais...]. Londrina, 2013. Disponível em: http://www.uel.br/ eventos/congressomultidisciplinar/pages/arquivos/anais/2013/AT03-2013/AT03-012.pdf Acesso em: 20 out. 2018.

RUST, Ryan; SINELNIKOV, Oleg Practicum in a self-contained environment: pre-service teacher perceptions of teaching students with disabilities. The Physical Educator, v. 1, n.1, 33-45, 2010.

SACRISTÁN, José G. 0 currículo: uma reflexão sobre a prática. 3. ed. Porto Alegre: Artmed, 2000.

SILVA, Cláudio Silvério da; DRIGO, Alexandre Janotta. Educação Física Adaptada: implicações curriculares e formação profissional. São Paulo: Cultura Acadêmica, 2012.

TALIAFERRO, Andrea R.; HAMMOND, Lindsay; WYANT, Kristi. Preservice Physical Educators' Self-Efficacy beliefs toward inclusion: the impact of coursework and practicum. Adapted Physical Activity Quarterly, v. 32, p. 49-67, 2015.

TINDALL, Daniel et al. Pre-service teachers' attitudes towards children with disabilities: an Irish perspective. European Physical Education Review, v. 21, n.2, e p. 206-221, 2014. 
VICKERMAN, Philip; COATES, Janine K. Trainee and recently qualified physical education teachers' perspectives on including children with special educational needs. Physical Education and Sport Pedagogy, v. 14, n. 2, p. 137-153, 2009.

WILKINSON, Shawn et al. Student teacher experiences in a service-learning project for children with attention-deficit hyperactivity disorder. Physical Education and Sport Pedagogy, v. 18, n. 5, p. 475-491, 2013.

WOODRUFF, Elizabeth A.; SINELNIKOV, Oleg A. Teaching young adults with disabilities through service learning. European Physical Education Review, v. 21, n.3, p. 292-308, dec. 2014.

Apoio:

O presente trabalho foi realizado com apoio da Coordenação de Aperfeiçoamento de Pessoal de Nível Superior Brasil (CAPES) - Código de Financiamento 001. This study was financed in part by the Coordenação de Aperfeiçoamento de Pessoal de Nível Superior - Brasil (CAPES) - Finance Code 001. 